\title{
Vulnérabilité de l'eau souterraine à la contamination par les nitrates sur le bassin versant de la rivière Noire (Montérégie, Québec)
}

\section{Groundwater vulnerability in the Noire River watershed (Montérégie, Québec)}

\author{
Sylvain Bolduc, Marie Larocque et Gilbert Prichonnet
}

Volume 19, numéro 2, 2006

URI : https://id.erudit.org/iderudit/013043ar

DOI : https://doi.org/10.7202/013043ar

\section{Aller au sommaire du numéro}

\section{Éditeur(s)}

Université du Québec - INRS-Eau, Terre et Environnement (INRS-ETE)

ISSN

1718-8598 (numérique)

Découvrir la revue

Citer cet article

Bolduc, S., Larocque, M. \& Prichonnet, G. (2006). Vulnérabilité de l'eau souterraine à la contamination par les nitrates sur le bassin versant de la rivière Noire (Montérégie, Québec). Revue des sciences de l'eau / Journal of Water Science, 19(2), 87-99. https://doi.org/10.7202/013043ar

\section{Résumé de l'article}

La rivière Yamaska est l'un des affluents du Saint-Laurent les plus contaminés par les activités agricoles. Cette problématique touche particulièrement le sous-bassin de la rivière Noire où les dépôts de surface du Quaternaire sont discontinus, de faible épaisseur et souvent perméables. L'objectif de cette étude est de déterminer la vulnérabilité de l'eau souterraine sur une partie du sous-bassin de la rivière Noire $\left(100 \mathrm{~km}^{2}\right)$. La méthodologie utilisée comprend la caractérisation de l'aquifère, l'analyse des concentrations en nitrates et des contenus en $\delta^{18} \mathrm{O}$, l'étude de la vulnérabilité en utilisant l'approche AQUIPRO et la modélisation hydrogéologique. Les résultats montrent une dégradation significative et d'origine anthropique de l'eau souterraine : plusieurs concentrations excèdent $1 \mathrm{mg} \mathrm{N}-\mathrm{NO}_{3} / \mathrm{L}$ et quelques-unes excèdent $10 \mathrm{mg}$ $\mathrm{N}-\mathrm{NO}_{3} / \mathrm{L}$. Les puits contaminés sont situés sur les crêtes topographiques où le silt argileux est absent et le till discontinu, et où la plus grande vulnérabilité AQUIPRO a été identifiée. Une diminution des concentrations avec la profondeur de prélèvement s'explique par un écoulement souterrain peu profond entraînant les nitrates vers un ruisseau et vers la rivière Noire. La vulnérabilité de l'eau souterraine est ainsi beaucoup plus grande dans les couches superficielles du roc fracturé. Le temps moyen de séjour de l'eau souterraine est estimé à 20 ans. Les concentrations mesurées permettent d'établir un lien direct entre la vulnérabilité, les dépôts de surface, la stratigraphie du substrat et les directions de l'écoulement souterrain. Elles démontrent aussi la présence d'une contamination de l'eau souterraine par les nitrates qui est susceptible d'augmenter si aucune mesure préventive n'est mise en place. 


\title{
VULNÉRABILITÉ DE L'EAU SOUTERRAINE À LA CONTAMINATION PAR LES NITRATES SUR LE BASSIN VERSANT DE LA RIVIÈRE NOIRE (MONTÉRÉGIE, QUÉBEC)
}

\author{
Groundwater vulnerability in the Noire River watershed (Montérégie, Québec)
}

\begin{abstract}
Sylvain Bolduc', Marie Larocque ${ }^{2 *}$ et Gilbert Prichonnet, ${ }^{23}$
${ }^{1}$ Nove Environnement, 1650, rue Champlain, Trois-Rivières (Québec) Canada G9A 4S9.,

${ }^{2}$ Département des Sciences de la Terre et de l'Atmosphère, Université du Québec à Montréal, Case Postale 8888, succursale Centre-Ville, Montréal (Québec) Canada H3C 3P8.

${ }^{3}$ GEOTERAP (Groupe de recherche en Géologie de Terrain Appliquée) Dép. Sciences de la Terre et de l'atmosphère, Université du Québec à Montréal, Case Postale 8888, succursale Centre-Ville, Montréal (Québec) Canada H3C 3P8.
\end{abstract}

Reçu le 16 décembre 2004, accepté le15 août 2005

\section{ABSTRACT}

Many studies have shown a link between intensive agricultural practices and groundwater pollution by nitrates. In Québec, recent studies have shown that the Yamaska River is highly contaminated by agricultural activities. Maize and pork production are particularly intensive in the Noire River subbasin. In this area, quaternary deposits are discontinuous, of limited thickness and are generally permeable, leading to high groundwater vulnerability. The objective of this study was to determine groundwater vulnerability to nitrate contamination on a small agricultural sub-watershed of the Noire River. The methodology included aquifer characterization, analysis of nitrate concentrations and $\delta^{18} \mathrm{O}$ composition, as well as a vulnerability evaluation and groundwater flow modelling.

Located on the south shore of the Saint Lawrence River, the Noire River $\left(1579 \mathrm{~km}^{2}\right)$ is located in the southeastern portion of the Yamaska basin. A small part of the basin $\left(100 \mathrm{~km}^{2}\right)$ was the focus of this study. The Noire River flows in the centre of the study area whereas the Aulnages creek is a small tributary to the Noire River. The study area was limited to the east and west by topographic highs. It is located between the limit of the St. Lawrence Lowlands and the first Appalachian ridges. The bedrock, Cambrian to Ordovician in age, is mainly made of limestone in the western zone and is composed of terrigenous siliciclastic facies in the eastern zone. The substratum forms elongated crests, due to the tectonic grain, surrounded by recent surface deposits. These quaternary deposits are discontinuous and are of limited thickness. The hydrological potential of the fractured rock aquifer is interesting but spatially variable.

The deposits were analyzed at 50 observation sites and 18 typical samples were sieved or submitted for density analysis (GEOTERAP). Data from the Système d'Information Hydrogéologique (SIH) were used to complete the field information in establishing the stratigraphy of the area. Soil hydraulic conductivity was measured using a Guelph permeameter and two short-term pumping tests were performed. Monthly water levels were measured in 18 private wells from June 2001 to June 2002. Water was sampled bimonthly from 35 sites ( 25 deep wells, two shallow wells, two sites in the Noire River, four in the Aulnages stream, and two in a temporary lake, located in a gravel pit). In October 2001

*Auteur pour correspondance :

Tél. : (514) 987-3000+1515

Fax : (514) 987-7749

Courriel : marie.larocque@uqam.ca 
and April 2002, water was sampled for $\delta^{18} \mathrm{O}$ composition. Analyses were performed at the GÉOTOP-UQÀM-McGill laboratories. Aquifer vulnerability was determined using the AQUIPRO approach, a simple method that considers clay and till thickness, in addition to well depth. A groundwater flow model was developed using MODFLOW and MODPATH to simulate groundwater flow, flow paths and residence times.

Characterization of the quaternary deposits confirmed the following sequence, from the base to the top: till; clayey silts; sand and sandy gravel. The thickness of these deposits was variable, and there were bedrock outcrops, mainly on the western and eastern sides of the study area. A north-south esker (partially exploited) is present on the western side of the Noire River. Measured soil hydraulic conductivities show that the clayey silts and till deposits have low permeability whereas the sand is more permeable. Clayey silts present in the centre of the study area, as well as occasional till deposits, offer partial protection to the aquifer, whereas elsewhere infiltration and contaminants can reach the aquifer through recharge. Pumping tests showed high transmissivities for the rock formation, probably resulting from the important rock fracturing in the upper 10 to $15 \mathrm{~m}$ of the aquifer. Groundwater flowed towards the river from the eastern and western limits of the study area, and water depth was on average $2.5 \mathrm{~m}$. The Noire River is generally in contact with rock and drains the aquifer.

Measured nitrate concentrations exceeded $10 \mathrm{mg}$ $\mathrm{N}-\mathrm{NO}_{3} / \mathrm{L}$ (guideline for potable water) in the two sampled surface wells. Concentrations were between 1 and $10 \mathrm{mg}$ $\mathrm{N}-\mathrm{NO}_{3} / \mathrm{L}$ at least once during the study period in ten of the 25 deep sampled wells, indicating a groundwater contamination problem of anthropogenic origin. The highest concentrations were measured in recharge areas and nitrate concentrations were found to decrease generally with well depth. Nitrate concentrations were higher in the Aulnages creek than in the Noire River, probably because the creek intercepts drainage water and shallow groundwater flow. The isotopic composition of surface wells, deep wells and Aulnages creek water remained relatively stable between sampling times. This indicates an important mixing of fresh recharge with in situ groundwater. In the Noire River, $\delta^{18} \mathrm{O}$ compositions reflect the yearly variations in precipitation.

AQUIPRO aquifer vulnerability was highest in the eastern and western parts of the study area where the highest nitrate concentrations were measured. AQUIPRO vulnerability was lowest where the clayey silts provide some protection near the Noire River. The results showed an important spatial heterogeneity in the thickness of the clayey silt layer, underlining the generally high aquifer vulnerability in the study region. The groundwater flow model developed using field data simulated the measured heads adequately. Flow directions simulated with MODPATH confirmed the presence of a shallow groundwater flow from the eastern and western limits and towards the Noire River. This flow is probably responsible for the decreasing nitrate concentrations observed with increasing water sampling depth and confirms that aquifer vulnerability varies with depth in the aquifer. The average groundwater residence time is 20 years. This means that recharge will take on average 20 years to travel through the aquifer and to the Noire River. It also indicates the time frame required for the aquifer to eliminate a large-scale nitrate contamination, after the initiation of control measures.

This work showed that groundwater nitrate contamination is related to groundwater vulnerability, which is a function of quaternary deposits, substrate stratigraphy and groundwater flow directions. These factors must be considered when studying groundwater vulnerability as they directly affect contaminant transport. It therefore appears necessary to use a combination of various approaches to better understand aquifer vulnerability and to design preventive measures. This work also demonstrated groundwater contamination by nitrates in the study region. Because of the generally high vulnerability of the aquifer, increased nitrate contamination can be expected in the future if no preventive measures are undertaken to protect the groundwater resource.

Key words: vulnerability, groundwater, nitrates, contamination, agriculture, AQUIPRO, groundwater modelling, Province of Quebec.

\section{RÉSUMÉ}

La rivière Yamaska est l'un des affluents du Saint-Laurent les plus contaminés par les activités agricoles. Cette problématique touche particulièrement le sous-bassin de la rivière Noire où les dépôts de surface du Quaternaire sont discontinus, de faible épaisseur et souvent perméables. L'objectif de cette étude est de déterminer la vulnérabilité de l'eau souterraine sur une partie du sous-bassin de la rivière Noire $\left(100 \mathrm{~km}^{2}\right)$. La méthodologie utilisée comprend la caractérisation de l'aquifère, l'analyse des concentrations en nitrates et des contenus en $\delta^{18} \mathrm{O}$, l'étude de la vulnérabilité en utilisant l'approche AQUIPRO et la modélisation hydrogéologique. Les résultats montrent une dégradation significative et d'origine anthropique de l'eau souterraine: plusieurs concentrations excèdent $1 \mathrm{mg}$ $\mathrm{N}-\mathrm{NO}_{3} / \mathrm{L}$ et quelques-unes excèdent $10 \mathrm{mg} \mathrm{N}-\mathrm{NO}_{3} / \mathrm{L}$. Les puits contaminés sont situés sur les crêtes topographiques où le silt argileux est absent et le till discontinu, et où la plus grande vulnérabilité AQUIPRO a été identifiée. Une diminution des concentrations avecla profondeur de prélèvements'explique par un écoulement souterrain peu profond entraînant les nitrates vers un ruisseau et vers la rivière Noire. La vulnérabilité de l'eau souterraine est ainsi beaucoup plus grande dans les couches 
superficielles du roc fracturé. Le temps moyen de séjour de l'eau souterraine est estimé à 20 ans. Les concentrations mesurées permettent d'établir un lien direct entre la vulnérabilité, les dépôts de surface, la stratigraphie du substrat et les directions de l'écoulement souterrain. Elles démontrent aussi la présence d'une contamination de l'eau souterraine par les nitrates qui est susceptible d'augmenter si aucune mesure préventive n'est mise en place.

Mots clés : vulnérabilité, eau souterraine, nitrates, contamination, agriculture, AQUIPRO, modélisation hydrogéologique, Québec.

\section{INTRODUCTION}

L'agriculture moderne favorise les cultures intensives et une utilisation abondante de fertilisants. Il y a cependant des risques associés à ces pratiques culturales puisque l'utilisation excessive des engrais chimiques et organiques peut entraîner le lessivage de nitrates vers l'eau souterraine (REFSGAARD et al., 1999). Partout dans le monde, des observations et des études indiquent un lien étroit entre l'agriculture intensive et les fortes concentrations en nitrates dans l'eau souterraine (PRIMEAU et GRIMARD, 1989; BERGSTRÖM et JARVIS, 1991; ANDREWS et al., 1997; LASSERRE et al., 1999; SIDD, 2003).

Au Québec, les pressions d'origine agricole sur la qualité de l'eau et sur l'environnement résultent principalement de la monoculture intensive du maïs et du développement d'élevages de grandes dimensions. Les superficies de culture du maïs ont augmenté de $29 \%$ de 1996 à 2001 (GIROUX, 2002), une hausse attribuable en partie à l'augmentation de la production porcine car le maïs-grain est utilisé pour l'alimentation des porcs. Les besoins élevés en fertilisants pour cette culture entraînent un épandage de quantités importantes de lisier. L'entreposage et l'épandage inadéquats des déjections animales constituent une source potentielle de pollution microbienne, organique et minérale ponctuelle ou diffuse (SAINT-LAURENT VISION 2000, 1999). Au Québec, la contamination des aquifères par les nitrates est une préoccupation constante pour l'environnement (e.g. eutrophisation des eaux de surface), et surtout parce que $23 \%$ de la population, dont une large majorité de la population rurale, consomment de l'eau souterraine provenant d'un puits individuel ou d'un réseau municipal alimenté par l'eau souterraine (MENV, 2004).

La rivière Yamaska est parmi les affluents du fleuve SaintLaurent les plus affectés par la pollution d'origine agricole. Le sous-bassin de la rivière Noire est particulièrement touché en raison de l'intensification de l'élevage porcin et de la culture du maïs (BAPE, 2000). Selon une étude de la vulnérabilité des formations aquifères à l'échelle régionale (McCORMACK et
LACOULINE, 1996), environ $77 \%$ du territoire du bassin de la rivière Noire serait vulnérable à la contamination de l'eau souterraine. Des cas localisés de contamination de l'eau souterraine (PARÉ, 1978; GAUDREAU et MERCIER, 1997) laissent croire que la vulnérabilité est très variable d'un site à l'autre. En effet, la présence de sédiments quaternaires, de composition, de texture et d'épaisseur inégales, conditionne souvent le potentiel de contamination variable de l'eau souterraine. Une étude hydrogéologique régionale a été réalisée sur le bassin de la Yamaska (PARÉ, 1978), mais aucune étude n'a été menée tant avec une maille serrée d'échantillonnage sur l'hydrogéologie, que pour étudier la vulnérabilité locale de l'eau souterraine.

Il existe plusieurs méthodes d'évaluation de la vulnérabilité de l'eau souterraine à la contamination. DRASTIC (ALLER et al., 1987) est un système de cotation numérique qui tient compte de sept facteurs hydrogéologiques pour déterminer un indice de vulnérabilité. AQUIPRO (CHOWDHURY et al., 2003) est aussi basé sur une cotation numérique, mais tient compte d'informations stratigraphiques seulement pour estimer un indice de vulnérabilité. L’approche développée par ROSS et al. (2004) évalue la vulnérabilité basée à la fois sur la stratigraphie, selon une vue tridimensionnelle du sous-sol, et sur le temps de transfert convectif vertical. Enfin, EVARISK (BANTON et al., 1998) simule la migration d'un contaminant d'origine diffuse dans l'eau lessivée au-delà de la zone racinaire et peut être utilisé pour estimer l'apport de contaminant à la nappe. Ces approches ont en commun qu'elles ne tiennent pas compte de l'écoulement souterrain régional dans leur évaluation de la vulnérabilité de l'aquifère. Pour considérer celui-ci, il faut utiliser des approches plus complexes basées sur la modélisation numérique de l'écoulement souterrain (e.g. ROBINSON et REAY, 2002) ou du transport de contaminant (e.g. REFSGAARD et al., 1999).

L'absence de connaissance hydrogéologique précise sur le bassin de la rivière Noire a motivé la réalisation d'une étude à l'échelle locale du contexte géologique et hydrogéologique, afin d'évaluer les risques de contamination de l'aquifère. La présente étude a pour objectif de déterminer les zones vulnérables à la contamination par les nitrates à l'échelle d'un sous-bassin versant très agricole de la rivière Noire. Elle se divise en quatre parties: la caractérisation géologique et hydrogéologique, l'analyse des contenus en nitrates et de la composition en isotopes de l'oxygène, le calcul de la vulnérabilité de l'aquifère, et enfin la simulation de l'écoulement.

\section{MATÉRIEL ET MÉTHODES}

\subsection{Description de la zone d'étude}

Située sur la rive sud du Saint-Laurent, la rivière Yamaska 
prend sa source dans le lac Brome et se jette dans le fleuve à la hauteur du lac Saint-Pierre. Le sous-bassin de la rivière Noire couvre une superficie de $1579 \mathrm{~km}^{2}$, au sud-est du bassin de la Yamaska (Figure 1). Afin d'étudier la vulnérabilité de l'aquifère à l'échelle locale, une portion seulement du bassin de la rivière Noire a été retenue pour cette étude. Cette zone couvre environ $100 \mathrm{~km}^{2}$, près de la confluence de la rivière Noire avec la Yamaska, entre les municipalités de Saint-Hyacinthe et Granby. La topographie varie entre $45 \mathrm{~m}$, au point le plus en aval de la rivière Noire, et $120 \mathrm{~m}$ pour les crêtes au sud-est de la zone d'étude. Sur ce territoire, deux cours d'eau coulent du nord vers le sud: la rivière Noire, située au centre de la zone, et le ruisseau des Aulnages, un tributaire de la rivière Noire. La zone d'étude correspond à l'ouest à la limite du sousbassin versant du ruisseau des Aulnages et à l'est à une crête topographique divisant l'écoulement entre la rivière Noire et un sous-bassin versant voisin.

La zone étudiée est située à la limite des Basses-Terres du Saint-Laurent et des premières crêtes appalachiennes. Le

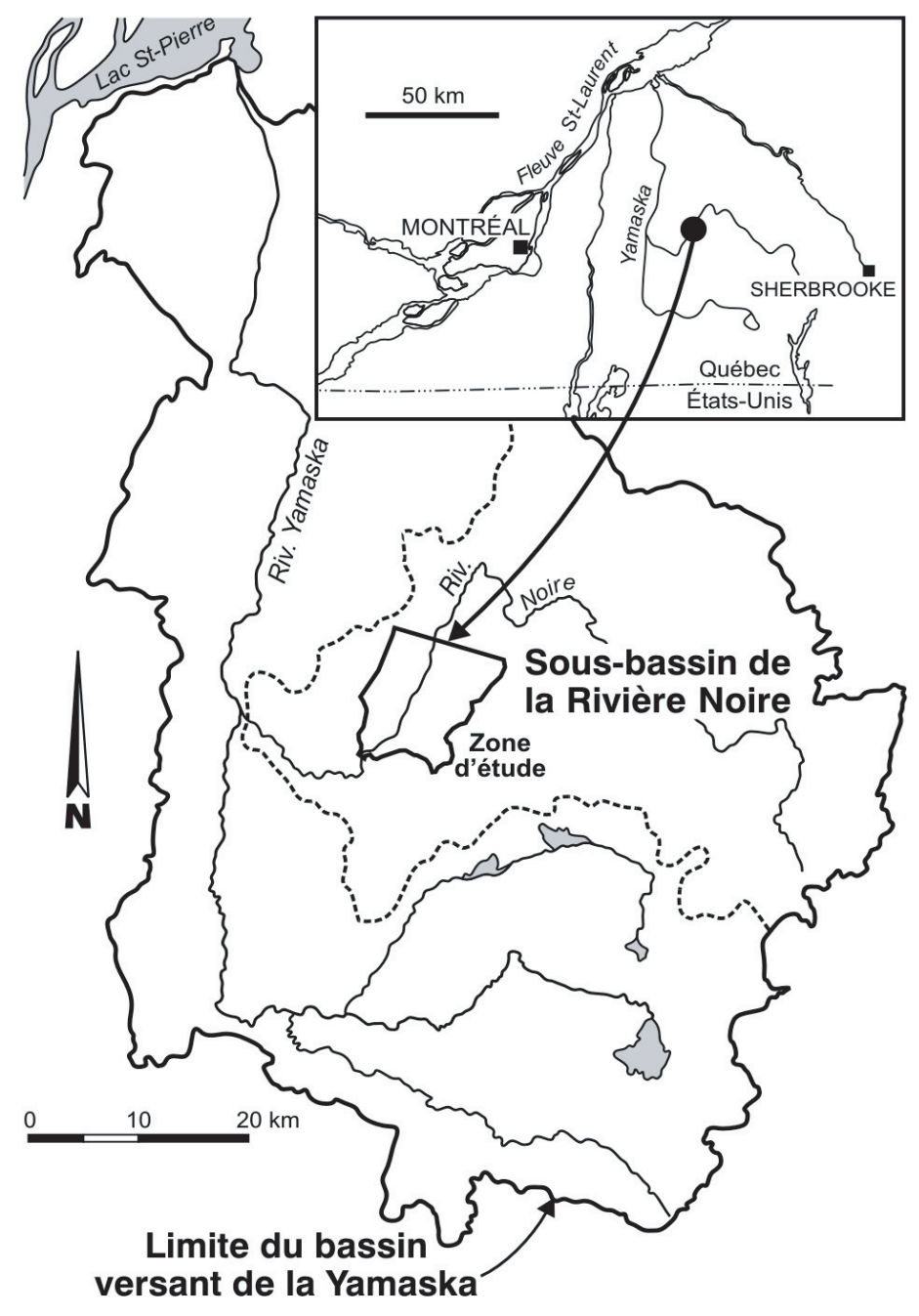

Figure 1 Localisation de la zone d'étude. Location of the study area. terrain s'étend du versant est de la crête de Saint-Dominique (une écaille de roches plissées) jusqu'aux limites orientales de la Nappe de Granby. Celle-ci, limitée à l'ouest par la Ligne Logan (une faille majeure de chevauchement), fait partie du domaine externe des Appalaches. Les roches du substratum sont carbonatées à l'ouest (calcaires, grès et conglomérats) et détritiques terrigènes (ardoises et schistes) à l'est (COOKE et al., 1962; CLARK, 1977). Les crêtes rocheuses, d'âge cambrien à ordovicien, forment des îlots allongés au milieu de la couverture de dépôts meubles récents (PRICHONNET, 1984). Le relief s'accentue sur le versant est de la rivière Noire car le substrat rocheux y est plus proche de la surface, et même souvent affleurant. Le paysage devient alors plus boisé qu'à l'ouest et au centre, zones où l'agriculture du maïs domine.

Pendant le Wisconsinien supérieur et le début de l'Holocène (GADD et al., 1972; PRICHONNET, 1977; PRICHONNET et al., 1982), des accumulations sédimentaires variées ont recouvert le piedmont et les premières crêtes pénéplanisées des Appalaches. Les dépôts meubles étant discontinus et d'épaisseur limitée, l'aquifère régional se situe dans le roc. Celui-ci est fracturé et parfois altéré en surface, ce qui rend son potentiel aquifère relativement intéressant mais assez variable.

Plusieurs types de sol ont été identifiés dans la zone d'étude, allant du gravier sableux au loam argileux, le plus souvent en association avec les dépôts de surface. Lagriculture couvre entre 70 et $75 \%$ du territoire. En 2001, le maïs occupait $65 \%$ des terres cultivées, le soya $17 \%$ et les prairies, pâturages et autres cultures $18 \%$ (POISSON, 2004).

\subsection{Méthodologie}

La caractérisation géologique des dépôts de surface d'origine glaciaire et post-glaciaire est basée sur l'interprétation de forages et l'analyse d'échantillons typiques des principaux faciès cartographiés dans le secteur. Les données de 118 forages extraits du Système d'Information Hydrogéologique (SIH) (MENV, 2005) ont été utilisées afin de contrôler l'épaisseur des dépôts de surface et la succession des unités stratigraphiques. L'étude des photographies aériennes au 1/15 000 a permis par ailleurs d'interpréter la distribution spatiale des dépôts meubles quaternaires, préalablement à leur échantillonnage. L'étude stratigraphique et sédimentologique s'appuie enfin sur une cinquantaine de sites d'observation des dépôts de surface et l'analyse de 18 échantillons (Figure 2). Elle inclut le tamisage des sédiments grossiers et la densimétrie des sédiments fins.

Des mesures mensuelles de niveaux piézométriques ont été réalisées dans 18 puits de particuliers de la zone d'étude de juin 2001 à juin 2002 (Figure 2). Les données extraites du $\mathrm{SIH}$ comprennent aussi des informations sur le niveau d'eau dans les forages, ceux-ci atteignant presque tous le roc. La conductivité hydraulique des sols a été mesurée à l'aide d'un 


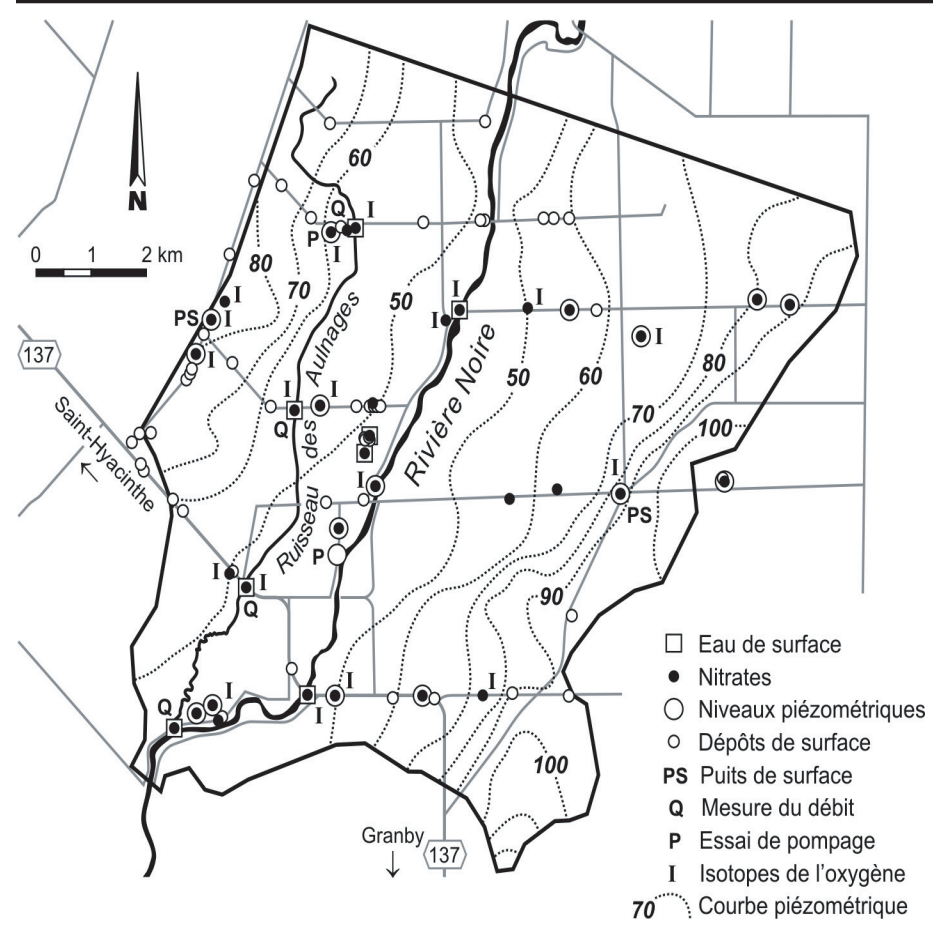

Figure 2 Stations d'échantillonnage et carte piézométrique. Sampling sites and piezometric map.

perméamètre de Guelph à $30 \mathrm{~cm}$ de profondeur pour 30 stations, de manière à la contrôler dans les différents types de sols du secteur. Deux essais de pompage de courte durée ont aussi été réalisés en juillet 2002 pour évaluer la conductivité hydraulique du roc. Les résultats de ces essais sont interprétés à l'aide de la méthode Cooper-Jacob (CHAPUIS, 1999).

Une campagne d'échantillonnage d'eau réalisée de juin 2001 à juin 2002 a permis de déterminer les teneurs en nitrates. Trente-cinq stations ont été échantillonnées tous les deux mois (Figure 2). Deux stations d'échantillonnage d'eau sont situées sur la rivière Noire, quatre sur le ruisseau des Aulnages, deux dans un lac temporaire (sablière localisée dans un esker partiellement exploité), et 27 dans des puits de particuliers, soit 25 puits profonds (au roc) et deux puits de surface (dépôts meubles). Les puits sont échantillonnés en amont de tout système de traitement. À chaque station, un échantillon de $4 \mathrm{ml}$ (volume standard requis et retenu pour l'analyse) a été prélevé et passé sur un filtre à $0,45 \mu \mathrm{m}$, puis conservé à $4{ }^{\circ} \mathrm{C}$ jusqu'à l'arrivée au laboratoire où il a été acidifié à l'acide sulfurique. En octobre 2001 et en avril 2002, les eaux de 14 puits, trois stations sur le ruisseau des Aulnages et deux sur la rivière Noire, ont été prélevées pour analyser leur composition en isotope lourd de l'oxygène $\left(\delta^{18} \mathrm{O}\right)$. Les concentrations en nitrates ont été mesurées suivant la méthode TRAACS 800 (méthode industrielle no 818-87T) et les isotopiques de l'oxygène ont été analysés par spectrométrie de masse au laboratoire GÉOTOP-UQÀM-McGill.

La vulnérabilité de l'aquifère a été évaluée à l'aide de la méthode AQUIPRO (CHOWDHURY et al., 2003). Cette méthode a été retenue car elle est très simple à appliquer et utilise les informations stratigraphiques disponibles dans la base de données du SIH, intégrant ainsi une information tridimensionnelle sur la nature des unités géologiques rencontrées. Les facteurs utilisés dans l'équation (1) sont l'épaisseur des couches d'argile et des couches de formations géologiques contenant de l'argile.

$$
A=D\left[3 \sum_{r=0}^{n}\left(\frac{C_{r}}{(1+r)}\right)+\sum_{r=0}^{n}\left(\frac{P_{r}}{(1+r)}\right)\right]
$$

où A est l'indice AQUIPRO, D est le coefficient de profondeur, C est l'épaisseur de l'argile (m), P est l'épaisseur des formations contenant de l'argile $(\mathrm{m})$ et $\mathrm{r}$ est le rang accordé à chaque couche. Pour un puits donné, les couches successives d'argile (et de formations géologiques contenant de l'argile) sont numérotées de la plus épaisse $(r=0)$ à la plus mince. Ceci permet d'accorder une importance plus grande aux couches les plus épaisses et généralement les plus étendues, qui assureront une plus grande protection de l'aquifère. Le facteur trois utilisé pour les couches d'argile accorde une plus grande importance à cette formation dans le calcul de la vulnérabilité. Le coefficient $\mathrm{D}$ varie de la valeur un pour un puits de moins de $2 \mathrm{~m}$ à la valeur huit pour un puits de plus de $183 \mathrm{~m}$, reflétant ainsi le fait que les puits profonds sont généralement moins vulnérables à la contamination. Un indice AQUIPRO élevé indique donc une vulnérabilité faible.

Finalement, un modèle hydrogéologique a été développé avec MODFLOW (McDONALD et HARBAUGH, 1988) pour simuler l'écoulement dans un milieu continu et en régime permanent. Le modèle tridimensionnel est composé de trente couches de cellules d'environ $200 \mathrm{~m}$ par $200 \mathrm{~m}$ de côté, par $3 \mathrm{~m}$ d'épaisseur. Les données géologiques et hydrogéologiques acquises sur le terrain et en laboratoire ont permis de définir et paramétrer le modèle tridimensionnel. La rivière Noire est représentée par une charge constante sur l'épaisseur d'une cellule tandis que le ruisseau des Aulnages n'est pas représenté en raison de son influence limitée sur l'écoulement souterrain. Les frontières est et ouest du modèle correspondent à des crêtes topographiques (également les limites de la zone d'étude), tandis que les frontières nord et sud sont situées sur des lignes de courant (limites à flux nul). Les paramètres ont été calés manuellement, de manière à reproduire les charges mesurées sur le terrain. Le logiciel MODPATH (POLLOCK, 1994), a ensuite été utilisé pour simuler les trajectoires d'écoulement et les temps de séjour de l'eau dans l'aquifère. 


\section{RÉSULTATS}

\subsection{Caractérisation géologique et hydrogéologique}

La caractérisation des différents faciès permet de retenir trois unités majeures de dépôts meubles reposant sur le substrat rocheux sous-jacent: le till, l'argile et le sable. Les analyses granulométriques ont montré que les matériaux fins sont en réalité du silt argileux (50\% de silt, $40 \%$ d'argile, et $10 \%$ de sable), mais cette composition peut varier, de la base, qui peut être plus argileuse, au sommet de l'unité. Les échantillons provenant des unités identifiées comme étant du sable ont révélé des textures très hétérogènes, incluant des graviers et parfois quelques blocs. Cette fraction grossière provient $\mathrm{du}$ remaniement du till qui est aussi l'assise stratigraphique régionale $\mathrm{du}$ Quaternaire. Les fractions fines du till ont été emportées par les courants et les vagues de la Mer de Champlain, puis déposées dans les zones profondes de plus faible énergie, particulièrement lors de l'exondation et à partir des zones rocheuses en relief. Ceci explique la présence des nombreux pointements rocheux au milieu des dépôts meubles en bordure du bassin versant. Le silt argileux correspond donc aux sédiments d'eaux profondes et les sables et graviers aux sédiments littoraux et sublittoraux (PRICHONNET, 1984). Le till original est toutefois présent sur une grande surface à l'affleurement dans l'est du bassin versant.

La figure 3 présente la carte géologique des dépôts meubles du secteur d'étude. Sur la coupe géologique, les trois unités de dépôts meubles reconnues sont représentées : des lentilles de till au contact du roc (dont l'extension pourrait être plus large, les données de forage étant assez imprécises à ce sujet); le silt argileux de la Mer de Champlain, qui constitue une couche imperméable au-dessus d'une nappe captive et qui peut localement reposer directement sur le roc; les sédiments granulaires (sables et graviers) sur les crêtes. Les roches du substratum rocheux sont non différenciées. Les dépôts de sable des zones en relief reposent souvent directement sur le roc, mais peuvent aussi succéder au silt argileux. L'épaisseur $\mathrm{du}$ silt argileux est très variable et atteint une valeur maximale de $20,4 \mathrm{~m}$ selon le SIH. L'épaisseur du sable ne dépasse généralement pas $6 \mathrm{~m}$, mais peut atteindre $35 \mathrm{~m}$ à l'aplomb de l'esker. Le till est d'épaisseur très variable, pouvant atteindre $14 \mathrm{~m}$ selon le $\mathrm{SIH}$, mais dépassant rarement $5 \mathrm{~m}$.

Les mesures de conductivité hydraulique des sols mesurées (Tableau 1) montrent que les sables constituent des dépôts poreux et perméables tandis que la conductivité hydraulique du silt argileux est faible. L'écart-type des conductivités hydrauliques mesurées dans le till montre que cette unité est de composition très variable et peut être relativement perméable par endroit et imperméable ailleurs. Les dépôts quaternaires vont donc contrôler les conditions de recharge de l'aquifère qui aura lieu surtout sur les crêtes topographiques où la couverture
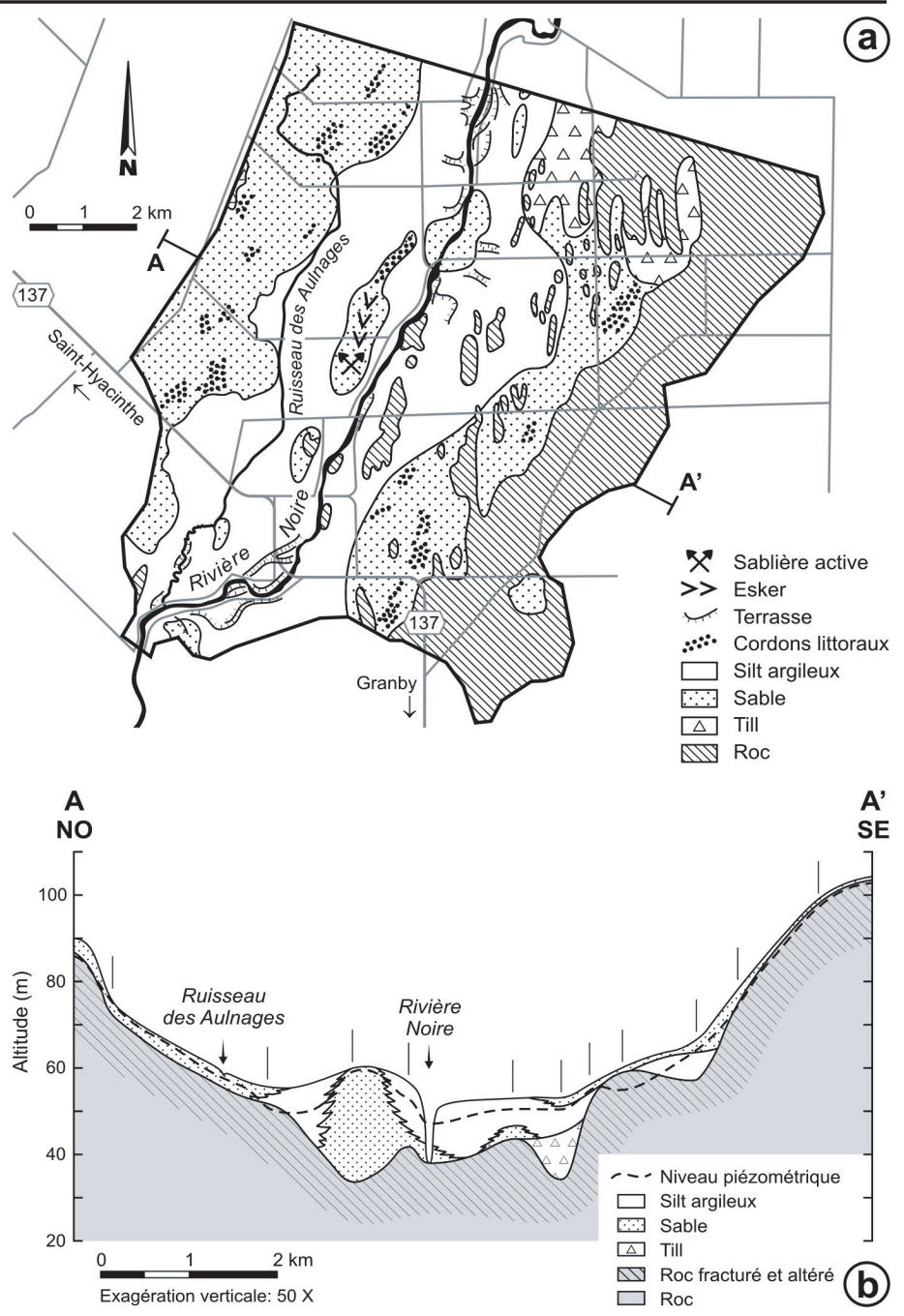

Figure 3 Géologie de surface a) carte géologique; b) coupe géologique.

Surface geology a) geological map; b) geological cross section.

de till est discontinue et où le roc affleure, sauf au nord-est du secteur où une couche de till plus continue a été identifiée. Les essais de pompage ont permis d'estimer la transmissivité $\mathrm{du}$ roc entre $2,5 \cdot 10^{-5}$ et $8,7 \cdot 10^{-5} \mathrm{~m}^{2} / \mathrm{s}$. Ces résultats sont $\mathrm{du}$ même ordre de grandeur que ceux de PARÉ (1978) obtenus sur 26 forages de la région. Les transmissivités relativement élevées pour ce type de roche (BANTON et BANGOY, 1999) pourraient provenir d'une fracturation importante et bien connectée partout sur le secteur d'étude. Une campagne géophysique par résistivité électrique (DJINENG NJOMO, 2002) a montré que les 10 à 15 premiers mètres du roc sont aussi les plus transmissifs. On interprète ce phénomène comme le résultat de la fracturation et de l'altération de la partie superficielle du roc résultant de la détente naturelle de la roche en surface et du lessivage par l'eau le long des fractures (diaclases principalement, et quelques failles). Ces facteurs pourraient faciliter l'écoulement souterrain peu profond des crêtes vers la rivière Noire. 
Tableau 1 Conductivités hydrauliques mesurées et calées.

Table 1 Measured and calibrated hydraulic conductivities.

\begin{tabular}{|c|c|c|c|}
\hline & \multicolumn{2}{|c|}{$\begin{array}{c}\text { Conductivité hydraulique } \\
(\mathrm{m} / \mathrm{s})\end{array}$} & $\begin{array}{c}\text { Porosité effective } \\
\text { (\%) }\end{array}$ \\
\hline Unité géologique & Valeurs mesurées & Valeurs calées & Littérature* \\
\hline Sable & $\begin{array}{c}\text { Moyenne }: 3,6.10^{-5}(\mathrm{n}=5) \\
\text { Écart-type }: 4,1.10^{-5}\end{array}$ & $5,0.10^{-4}$ & 30 \\
\hline Silt argileux & $\begin{array}{c}\text { Moyenne }: 1,2.10^{-6}(\mathrm{n}=13) \\
\text { Écart-type }: 2,1.10^{-6}\end{array}$ & $1,2.10^{-5}$ & 3 \\
\hline Till & $\begin{array}{c}\text { Moyenne: } 7,0.10^{-5}(\mathrm{n}=3) \\
\text { Écart-type }: 1,2.10^{-4}\end{array}$ & $1.10^{-6}$ & 5 \\
\hline Roc & $\begin{array}{c}\text { Minimum }: 2,5.10^{-6} * *(\mathrm{n}=2) \\
\text { Maximum }: 8,7.10^{-6}\end{array}$ & $\begin{array}{c}\text { Minimum }: 1.10^{-8} \\
\text { Maximum }: 5.10^{-4}\end{array}$ & 9 \\
\hline
\end{tabular}

* Valeurs tirées de BANTON et BANGOY (1997).

** Les conductivités hydrauliques sont estimées à partir des transmissivités mesurées par essai de pompage en supposant que l'aquifère a été sollicité sur une épaisseur de $10 \mathrm{~m}$.

Les niveaux piézométriques mesurés ont été relativement stables au cours de l'étude. Le niveau moyen a donc été utilisé avec les données issues du SIH afin de tracer une carte piézométrique de la zone d'étude (Figure 2). Celle-ci montre que le sens général de l'écoulement souterrain se fait vers la rivière Noire, avec un gradient hydraulique d'environ 0,01 dans le secteur ouest à 0,02 dans le secteur est. Le niveau piézométrique se situe en moyenne à $2,5 \mathrm{~m}$ sous la surface. La nappe, qui suit la topographie, est libre sur les crêtes et captive près de la rivière Noire, sous le silt argileux. La rivière Noire peut néanmoins être en contact avec le roc et drainer la nappe. Le ruisseau des Aulnages n'influence pas le niveau de la nappe, mais capte les drains agricoles et sans doute une partie de l'écoulement souterrain peu profond.

\subsection{Contenus en nitrates et composition en $\delta^{18} \mathrm{O}$}

Les concentrations en nitrates mesurées sur l'ensemble du territoire varient de 0 à $21,7 \mathrm{mg} \mathrm{N}-\mathrm{NO}_{3} / \mathrm{L}$ (Figure 4). Dix-sept (17) puits sur les 27 étudiés ont montré des concentrations inférieures à $1 \mathrm{mg} \mathrm{N}-\mathrm{NO}_{3} / \mathrm{L}$ pendant toute la période d'étude. Quatre puits profonds ont eu des concentrations comprises entre 1 et $3 \mathrm{mg} \mathrm{N}-\mathrm{NO}_{3} / \mathrm{L}$, et quatre des concentrations entre 3 et $10 \mathrm{mg} \mathrm{N}-\mathrm{NO}_{3} / \mathrm{L}$. Aucun puits profond n'a excédé la norme pour l'eau potable (10 mg N-NO $/ \mathrm{L}, \mathrm{AAC}, 2003)$ dans la zone d'étude. Mais les deux puits de surface ont montré des concentrations supérieures à $10 \mathrm{mg} \mathrm{N}-\mathrm{NO}_{3} / \mathrm{L}$ au moins une fois. Un puits profond situé dans une zone de recharge à proximité immédiate du secteur d'étude (non illustré à la Figure 4) a montré une concentration maximale de $35,8 \mathrm{mg}$ $\mathrm{N}-\mathrm{NO}_{3} / \mathrm{L}$ durant la même période de contrôle, ce qui confirme que la problématique de contamination s'étend en dehors de la zone étudiée. Les concentrations sont plus variables dans le temps pour les puits de surface que pour les puits profonds et aucune tendance à la hausse n'a été mise en évidence pendant la période d'étude. Les concentrations mesurées dans cette étude sont similaires à celles trouvées par PARÉ (1978)

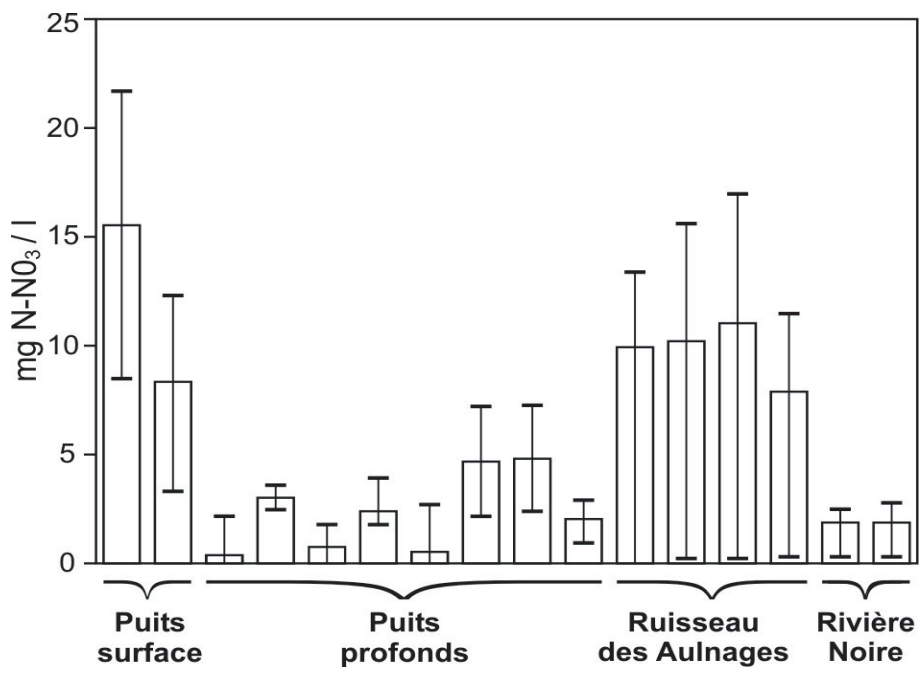

Figure 4

Concentrations en nitrates supérieures à $1 \mathrm{mg} \mathrm{N}-\mathrm{NO}_{3} / \mathrm{L}$ mesurées dans l'eau souterraine et de surface (rectangles : concentrations moyennes; barres: minimums et maximums).

Measured nitrate concentrations above $1 \mathrm{mg}$ $\mathrm{N}-\mathrm{NO}_{3} / \mathrm{L}$ in groundwater and surface water (rectangles: average concentrations; bars: minimum and maximum concentrations).

et GAUDREAU et MERCIER (1997) à l'échelle régionale. Elles sont aussi similaires à celles obtenues par le ministère de l'Environnement du Québec sur sept bassins versants du Québec en surplus de fumier (MENV, 2004).

Les concentrations en nitrates dans le ruisseau des Aulnages (Figure 4) sont souvent supérieures à $10 \mathrm{mg} \mathrm{N}-\mathrm{NO}_{3} / \mathrm{L}$ et très variables dans le temps. Dans la rivière Noire, les concentrations sont toujours inférieures à $3 \mathrm{mg} \mathrm{N}-\mathrm{NO}_{3} / \mathrm{L}$ et varient peu. Les concentrations plus élevées et plus variables dans le ruisseau des Aulnages proviennent sans doute du fait qu'il est alimenté en partie par de l'eau issue du drainage agricole qui circule rapidement à travers le sol superficiel riche en éléments 
nutritifs, entraînant avec elle les éléments solubles. Ce ruisseau intercepte aussi l'écoulement souterrain peu profond et plus contaminé. Les débits beaucoup plus grands dans la rivière Noire, ainsi que la contribution de l'eau souterraine peu contaminée contribuent à y maintenir les concentrations à des niveaux plus bas. Aucune tendance à la hausse dans le temps ou de l'amont vers l'aval n'a été mise en évidence pour ces cours d'eau. Les concentrations mesurées dans le lac situé dans l'esker sont inférieures à $1 \mathrm{mg} \mathrm{N}-\mathrm{NO}_{3} / \mathrm{L}$, indiquant que l'alimentation de ce lac provient des précipitations directes et de l'eau souterraine profonde non contaminée, à ce jour du moins.

La figure 5 (qui inclut les mesures répétées à une même station) illustre que les concentrations les plus élevées ont été mesurées dans les puits de surface (localisés dans les dépôts meubles) et dans le ruisseau des Aulnages. Cette relation entre la concentration et la profondeur des puits a été rapportée dans d'autres études (e.g. HUDAK, 2000). Dans les stations peu profondes, l'eau échantillonnée provient des couches superficielles de l'aquifère où les nitrates issus de la zone non saturée n'ont pas été fortement dilués. Dans un puits profond, l'eau prélevée provient d'une grande épaisseur d'aquifère et les nitrates issus de la surface sont plus dilués. Cette relation inverse entre les concentrations en nitrates et la profondeur des puits pourrait ne pas être valide partout, comme le démontre la présence mentionnée plus haut d'un puits profond fortement contaminé à proximité de la zone d'étude.

Les concentrations les plus élevées dans l'eau souterraine se retrouvent surtout dans les secteurs non protégés par le silt argileux, le long des crêtes rocheuses et des nappes sableuses de surface à l'est et à l'ouest de la zone d'étude (Figure 6). Les hétérogénéités des dépôts meubles sont donc à rechercher avec soin, car elles sont responsables des points de contamination

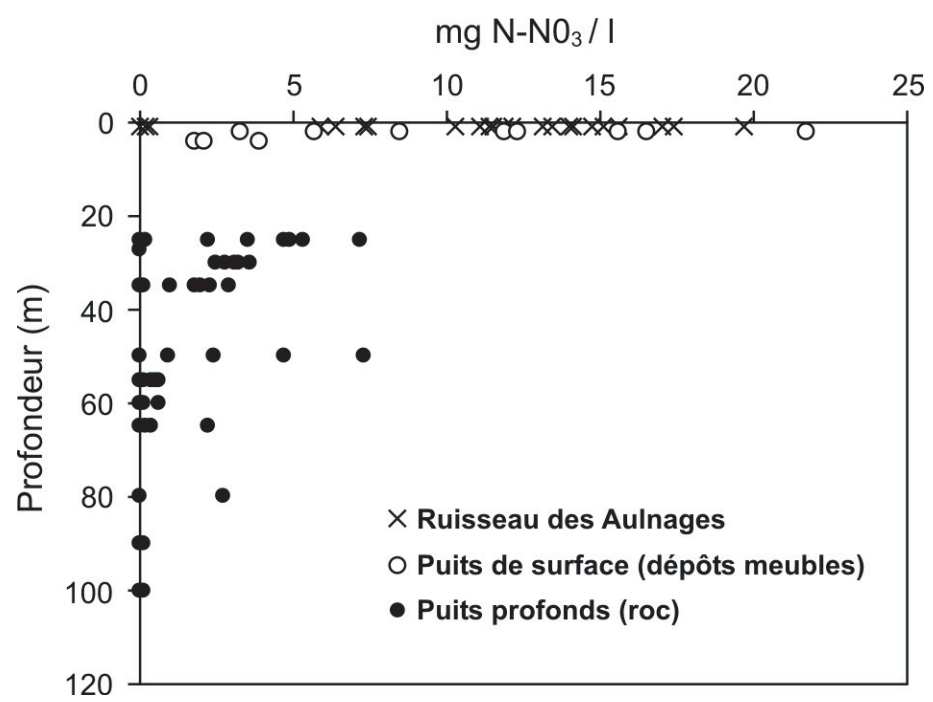

Figure 5 Lien entre la profondeur des forages et les concentrations en nitrates.

Relationshipbetween welldepthandnitrateconcentrations.

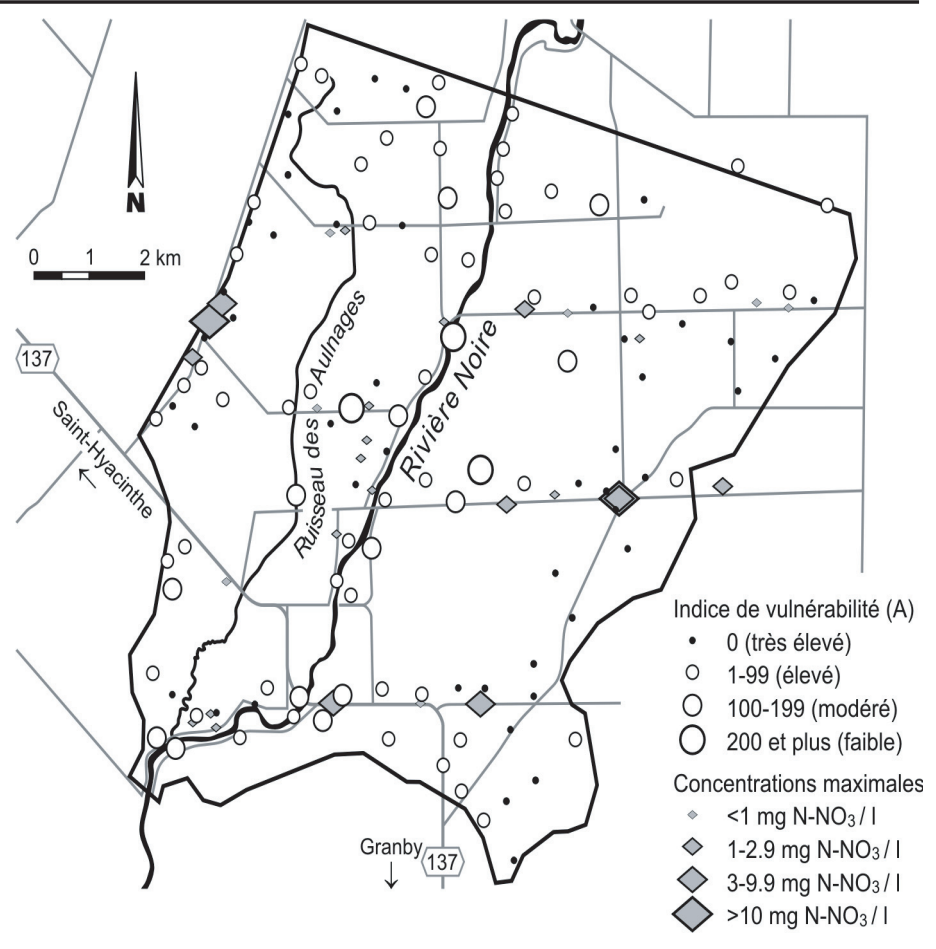

Figure 6 Concentrations maximales en nitrates dans l'eau souterraine et vulnérabilité AQUIPRO.

Maximum nitrate concentrations in groundwater and AQUIPRO vulnerability .

locaux. Les puits profonds situés près de la rivière Noire, prélevant l'eau sous le silt argileux et ne recevant pas directement la recharge contaminée, ont des concentrations généralement faibles, indiquant que l'eau chargée de nitrates provenant des zones amont subit une dilution importante lors de son écoulement dans l'aquifère. Un puits, situé dans le sud de la zone d'étude, dans une zone de silt argileux, fait toutefois exception avec une concentration maximale de 3,6 mg $\mathrm{N}-\mathrm{NO}_{3} / \mathrm{L}$. La protection offerte par les dépôts de surface n'est apparemment pas suffisante pour protéger complètement ce forage.

Même si elle n'est pas encore généralisée, comme c'est le cas dans d'autres régions d'agriculture intensive (e.g. LASSERRE et al.,1999; HUDAK, 2000), la contamination de l'eau souterraine par les nitrates est néanmoins présente dans la zone d'étude. Et malgré le fait que peu de puits excèdent la norme pour l'eau potable, les contenus en nitrates mesurés reflètent une contamination puisque les concentrations excèdent régulièrement $1 \mathrm{mg} \mathrm{N}-\mathrm{NO}_{3} / \mathrm{L}$, valeur considérée comme révélatrice d'un effet anthropique.

La composition moyenne en isotope de l'oxygène $\left(\delta^{18} \mathrm{O}\right)$ des puits profonds (Tableau 2) est sensiblement la même à l'automne 2001 et au printemps 2002. Ceci s'explique par un important mélange de la recharge avec l'eau souterraine, reflétant ainsi la composition moyenne des précipitations plutôt que celle de la recharge printanière appauvrie en $\delta^{18} \mathrm{O}$ 
Tableau 2 Composition moyenne en $\delta^{18} \mathrm{O}$ de l'eau souterraine et de surface.

Table 2 Average measured $\delta^{18} \mathrm{O}$ in groundwater and surface water.

\begin{tabular}{|l|c|c|}
\hline & \multicolumn{2}{|c|}{ Composition moyenne en $\boldsymbol{\delta}^{\mathbf{1 8}} \mathbf{O} \mathbf{( \% )}$} \\
\hline & Octobre 2001 & Avril 2002 \\
\hline Puits profonds $(\mathrm{n}=\mathbf{1 4})$ & $-10,6$ & $-10,5$ \\
\hline Puits de surface $(\mathrm{n}=\mathbf{2})$ & $-10,9$ & $-11,3$ \\
\hline Rivière Noire $(\mathrm{n}=\mathbf{2})$ & $-7,4$ & $-11,7$ \\
\hline Ruisseau des Aulnages $(\mathrm{n}=3)$ & $-10,5$ & $-10,7$ \\
\hline
\end{tabular}

(CLARK et FRITZ, 1997). La composition en $\delta^{18} \mathrm{O}$ des puits de surface semble légèrement plus appauvrie que celle des puits profonds et semble refléter celle de la recharge printanière, mais le nombre de stations de mesure $(\mathrm{n}=2)$ ne permet pas de le confirmer. La composition en $\boldsymbol{\delta}^{18} \mathrm{O}$ de l'eau de la rivière Noire varie selon la période de l'année tandis que celle du ruisseau des Aulnages est sensiblement constante et similaire à la composition moyenne de l'eau souterraine. La composition printanière appauvrie observée dans la rivière Noire s'explique par la fonte de la neige (récente en avril 2002); celle plus enrichie de l'automne est due à l'évaporation. La constance de la composition isotopique de l'eau du ruisseau des Aulnages reflète celle de l'eau souterraine d'où il tire donc une partie importante de son alimentation.

\subsection{Calcul d'un indice de vulnérabilité}

Dans cette section, la vulnérabilité de l'aquifere est quantifiée à l'aide de la méthode AQUIPRO. Les 118 forages extraits du SIH ont été utilisés pour calculer les indices de vulnérabilité dans la zone d'étude. Dans cette application de la méthode, le silt argileux est considéré comme de l'argile tandis que le till est considéré comme une formation contenant de l'argile. Puisque les forages ne présentent pas plus d'une couche de silt argileux ou de till, l'équation (1) se trouve simplifiée (voir équation (1) pour symboles) :

$$
\mathrm{A}=\mathrm{D}(3 \mathrm{C}+\mathrm{P})
$$

Un classement a été assigné à chaque indice pour illustrer la vulnérabilité relative de l'aquifere, tel que suggéré par CHOWDHURY et al. (2003) : vulnérabilité très élevée pour $A=0$; vulnérabilité élevée pour $1 \leq A \leq 99$; vulnérabilité modérée pour $100 \leq A \leq 199$; vulnérabilité faible pour $A \geq 200$. Dans la zone d'étude, les indices de vulnérabilité varient de 0 , où il $\mathrm{y}$ a absence de silt argileux et de till, à 317 pour un puits profond où le silt argileux est épais (Figure 6). Selon ce classement, trois puits seulement ont une vulnérabilité faible et 14 puits ont une vulnérabilité modérée. Tous sont situés à proximité de la rivière Noire, où le silt argileux est présent.
Parmi les autres puits, 54 présentent une vulnérabilité élevée et 47 ont une vulnérabilité très élevée. Ces derniers sont situés principalement sur les crêtes topographiques est et ouest, où il y a affleurement de roc, dépôts de sable sur roc ou encore des lentilles de till de faibles épaisseurs. La méthode AQUIPRO identifie aussi des puits très vulnérables près de la rivière Noire où les données de forage rapportent l'absence locale de silt argileux, malgré la couverture relativement continue décrite précédemment. Ceci souligne l'hétérogénéité spatiale du silt argileux et met en évidence la grande vulnérabilité de l'aquifère dans l'ensemble de la zone d'étude.

Les secteurs les plus vulnérables identifiés par la méthode AQUIPRO sur les crêtes topographiques sont aussi ceux où les concentrations en nitrates les plus élevées ont été observées (Figure 6). À l'exception d'un forage, tous les puits où une concentration supérieure à $1 \mathrm{mg} \mathrm{N}-\mathrm{NO}_{3} / \mathrm{L}$ a été mesurée se trouvent dans des secteurs à vulnérabilité très élevée ou élevée. Ceci confirme la pertinence de la méthode AQUIPRO et de la prise en compte de la stratigraphie pour identifier les zones les plus vulnérables à la contamination. Le puits faisant exception se trouve dans le sud de la zone d'étude où la vulnérabilité est modérée et où des dépôts de silt argileux ont été observés sur le terrain (Figure 2). Un défaut de conception (MAJDOUB et al., 2004), ou un apport d'eau souterraine contaminée provenant de l'amont, pourrait être à l'origine des concentrations élevées mesurées dans ce puits.

\subsection{Simulation de l'écoulement}

Le modèle conceptuel d'écoulement souterrain est basé sur les observations géologiques et hydrogéologiques présentées précédemment (une description exhaustive du modèle est disponible dans BOLDUC, 2004). La caractérisation géologique a permis de déterminer que les zones de recharge sont principalement situées sur les crêtes topographiques où le silt argileux est absent, la couverture de till discontinue et où le roc affleure, ou encore lorsque le roc est recouvert de sable. Dans le modèle hydrogéologique, une recharge moyenne de

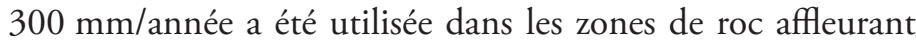
ou couvertes de sable, soit une valeur qui est typique pour une nappe libre du Québec méridional. La carte piézométrique a permis de confirmer que la rivière Noire agit comme un drain sur l'aquifère tandis que le ruisseau des Aulnages ne contrôle pas significativement l'écoulement souterrain. La rivière Noire a donc été représentée dans le modèle par une charge constante sur l'épaisseur d'une cellule, tandis que le ruisseau des Aulnages n'est pas pris en compte. La définition du modèle en 30 couches de $3 \mathrm{~m}$ chacune permet d'inclure la présence, démontrée par la géophysique, de roc plus fortement fracturé et altéré en surface.

Les conductivités hydrauliques ont été calées (Taleau 1) de manière à reproduire les charges mesurées, en tenant compte 
des valeurs obtenues sur le terrain et des résultats de PARÉ (1978). Des conductivités hydrauliques plus faibles ont été utilisées dans les couches sous-jacentes aux 10 à 15 premiers mètres de l'aquifère, permettant ainsi de simuler des charges élevées près des limites est et ouest du bassin. Des apports latéraux de faible importance, justifiés par la non-concordance entre les limites des bassins versants de surface et souterrains, ont aussi été utilisés à cette fin. Les différences entre les conductivités hydrauliques mesurées et calées pour les dépôts meubles s'expliquent par la grande hétérogénéité des dépôts de surface sur le terrain. La variabilité spatiale des conductivités calées pour l'unité de roc reflète le fait que le substratum est composé de plusieurs types de roches sédimentaires (calcaires, grès, ardoises, schistes et conglomérats) ayant un potentiel aquifère très variable. Le modèle calé simule correctement les niveaux piézométriques mesurés au cours de l'étude (Figure 7) avec une erreur moyenne sur les charges de $1,5 \mathrm{~m}$ (jugée acceptable car aucun puits n'a été nivelé). Ce calage confirme la pertinence du modèle conceptuel utilisé.

Le logiciel MODPATH a permis de simuler les trajectoires d'écoulement et le temps de transfert de l'eau souterraine. Une porosité effective moyenne issue de la littérature (BANTON et BANGOY, 1997) a été utilisée pour le calcul des vitesses réelles d'écoulement. Les trajectoires ont été tracées à partir de zones de roc affleurant ou de la succession sable sur roc. Elles montrent qu'un écoulement souterrain peu profond sur

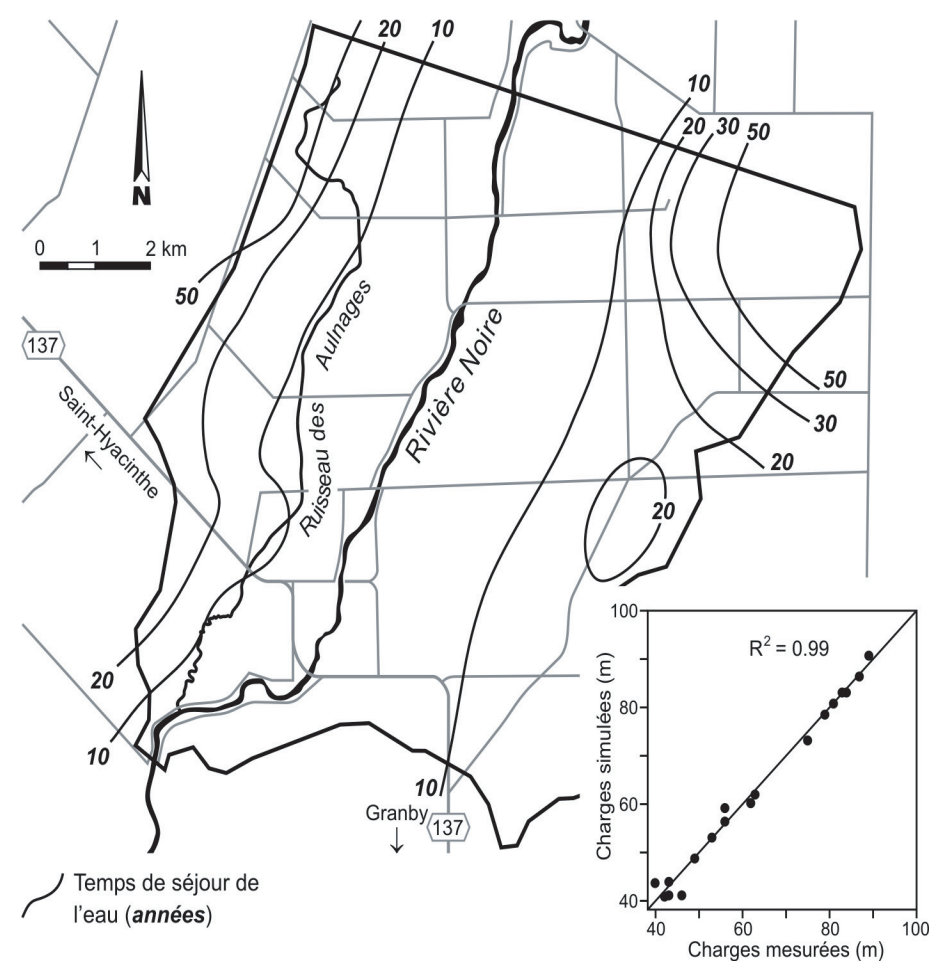

Figure 7 Charges hydrauliques calées et temps de séjour de l'eau souterraine.

Calibrated hydraulic heads and groundwater residence times. les crêtes topographiques entraîne les nitrates issus de la zone non saturée vers la rivière Noire.

Le temps de séjour moyen de l'eau dans l'aquifère varie de trois à 100 ans, et il est en moyenne de 20 ans entre les zones de recharge et la rivière Noire (Figure 7). Les temps les plus courts sont situés près de la rivière Noire et les plus élevés sont observés au nord-est de la zone d'étude, où une couche de till plus continue a été identifiée (Figure 3). C'est dire que la contamination actuelle résulte des pratiques agricoles réalisées en moyenne dans les 20 dernières années. Ces résultats identifient aussi l'intervalle de temps nécessaire pour qu'un changement au niveau des apports de nitrates depuis la surface se traduise par une diminution des concentrations dans l'eau souterraine. Cette période est du même ordre de grandeur que celle simulée par ROBINSON et REAY (2002) dans un aquifère de dépôts meubles de la Virginie. Certains choix issus de la définition même du modèle influencent directement les temps de séjour simulés. C'est le cas notamment de la porosité effective (estimée), de la combinaison conductivités hydrauliques (calées) - recharge (estimée), ainsi que du nombre de couches utilisé dans le modèle.

\section{DISCUSSION ET CONCLUSION}

Les résultats de cette étude montrent une dégradation significative d'origine anthropique de la qualité de l'eau souterraine dans la zone étudiée, même si les concentrations sont majoritairement en-deçà de la norme pour l'eau potable. Les puits contaminés sont généralement situés sur les crêtes topographiques où le silt argileux est absent et le till discontinu, et où la plus grande vulnérabilité AQUIPRO a été identifiée. La plupart des forages puisant leur eau sous le silt argileux et situés dans des zones de faible vulnérabilité AQUIPRO ne sont pas contaminés par les nitrates, en raison de l'absence d'une recharge directe à cet endroit et d'une dilution des polluants provenant de l'amont. Ces puits reçoivent néanmoins de l'eau contaminée de l'amont et, à plus ou moins long terme, selon la vitesse réelle de l'écoulement dans l'aquifère, la proximité des zones de recharge et l'évolution temporelle des apports de nitrates depuis la surface, la dilution pourrait ne plus suffire à maintenir les concentrations à des niveaux faibles. Il y aurait alors un risque de contamination pour l'ensemble de l'aquifère. C'est peut-être ce qui se produit déjà dans le puits où des concentrations élevées ont été observées au sud de la zone d'étude.

L'observation d'une diminution des concentrations avec la profondeur de prélèvement de l'eau s'explique par un écoulement souterrain peu profond, aussi mis en évidence par la modélisation, qui entraîne les nitrates vers la rivière Noire dans une trajectoire d'une durée moyenne de 20 ans. Ainsi, une zone identifiée comme très vulnérable par la cartographie 
des dépôts de surface, ainsi que par la méthode AQUIPRO, pourrait être peu vulnérable en profondeur (du moins à court terme) puisqu'un forage au roc n'y recevrait pas ou peu de recharge contaminée en raison d'un écoulement latéral vers la rivière Noire. Il n'est cependant pas exclu qu'un tel forage soit contaminé à la faveur de fractures transportant rapidement la recharge contaminée vers le bas, comme c'est déjà le cas pour certains puits situés sur les crêtes topographiques.

D'autres travaux seraient utiles afin d'apporter des précisions aux observations réalisées dans cette étude. Par exemple, la mesure du rapport isotopique ${ }^{15} \mathrm{~N} /{ }^{14} \mathrm{~N}$ dans l'eau permettrait d'identifier les sources ponctuelles et diffuses de la contamination, car les nitrates d'origine chimique, animale ou humaine, se distinguent par leur signature isotopique. Des travaux de datation de l'eau souterraine et des traçages permettraient aussi de confirmer les temps de séjour de l'eau dans l'aquifère (KATZ et al., 2004). Également, une analyse plus approfondie des pratiques agricoles locales permettrait de connaître les apports d'azote au sol et de quantifier les flux de nitrates hors de la zone racinaire (LAROCQUE et al., 2002). Enfin, une simulation de l'écoulement et du transport du sol à la nappe permettrait d'établir un lien entre l'occupation du territoire et la contamination de l'eau souterraine (LASSERRE et al., 1999).

En plus de la mise en évidence d'une vulnérabilité généralement élevée sur l'ensemble de la zone étudiée, les concentrations mesurées ont permis d'établir un lien direct entre la vulnérabilité et la nature des dépôts de surface, la stratigraphie et les directions d'écoulement souterrain. Ces facteurs influencent directement la migration des polluants dans l'eau souterraine, mais sont rarement pris en compte ensemble dans une évaluation globale du risque de contamination d'un aquifere. Lapplication successive de différentes approches d'évaluation de la vulnérabilité est donc absolument nécessaire pour bien comprendre les risques de contamination de l'eau souterraine et mettre en place des mesures préventives adéquates.

\section{REMERCIEMENTS}

Les auteurs remercient le FQRNT ainsi que l'UQÀM pour leur support financier. Ils remercient aussi M. G. Poisson (MAPAQ) ainsi que V. Delage et P. Benoit (COGEBY) pour avoir facilité le contact avec les résidents et fourni certaines données nécessaires à la réalisation de ce travail. Nos remerciements vont aussi à C. Chartrand, C. Dumont, G.T. Jineng Njomo, D. Fontaine, M. Laithier, M.C. Pharand et Y. Podkhlebnik du département des Sciences de la Terre et de l'Atmosphère de l'UQÀM pour leur support scientifique et technique. Finalement, les auteurs désirent remercier tout particulièrement les producteurs agricoles et les résidents ayant donné accès à leur propriété et permis d'analyser leur eau.

\section{RÉFÉRENCES BIBLIOGRAPHIQUES}

AAC (Agriculture et Agroalimentaire Canada), 2003. La santé de l'eau: Vers une agriculture durable au Canada. (Page web), Disponible à res2.agr.gc.ca/publications/hw/04c_ f.htm, Consultation en 2003.

ALLER L., BENNETT T., LEHR J.H., PETTY R. et HACKET G., 1987. A standardized system for evaluating ground water pollution potential using hydrogeological settings. US EPA Report EPA-600/2-87-035, Dublin (Ohio) et Ada (Oklahoma), États-Unis.

ANDREWS R. J., LLOYD J. W., et LERNER D. N., 1997. Modelling of nitrate leaching from arable land into unsaturated soil and chalk: 1. Development of a management model for applications of sewage sludge and fertilizer. J. Hydrol., 200, 179-197.

BANTON O., GOSSELIN M.A. et LAROCQUE, M., 1998. EvaRisk 1.0 - Manuel d'utilisation. Logiciel d'évaluation des risques de contamination des eaux souterraines par les sources diffuses. Projet réalisé pour le ministère de l'Environnement du Québec. INRS-Eau, Sainte-Foy, Québec, Rapport scientifique.

BANTON O. et BANGOY L. M., 1997. Hydrogéologie Multiscience environnementale des eaux souterraines: Québec. Presses de l'Université du Québec - AUPELFUREF, Québec, Canada.

BAPE, 2000. L'eau, ressource à protéger, à partager et à mettre en valeur. Rapport de la Commission sur la gestion de l'eau au Québec, Bureau d'audiences publiques sur l'environnement, Québec, Canada.

BERGSTRÖM L. et JARVIS N. J. 1991. Prediction of nitrate leaching losses from arable land under different fertilization intensities using the SOIL-SOILN models. Soil Use Manage., 7, 79-85.

BOLDUC S., 2004. Vulnérabilité de l'eau souterraine à la contamination par les nitrates: géologie, hydrogéologie et simulation sur le bassin versant de la rivière Noire (Montérégie, Québec). Mémoire de Maîtrise en Sciences de la Terre, Université du Québec à Montréal, Montréal, Canada, 156 p.

CHAPUIS R.P.,1999. Guide des essais de pompage et leurs interprétations. Les Publications du Québec, Sainte-Foy, Québec, Canada.

CHOWDHURY S.H., KEHEW A.E. et PASSERO R., 2003. 
Correlation between nitrate contamination and ground water pollution potentials. Ground Water, 41, 735-745.

CLARKT.H., 1977. Région de Granby (W); rapportgéologique 177. Carte 1869, 1:63 360. Service de l'exploration géologique, direction générale des mines, Ministère des Richesses naturelles, Québec, Canada, 109 p.

CLARK I.D. ET FRITZ P., 1997. Environmental isotopes in hydrogeology. LEWIS Publishers, New York, USA.

COOKE H.C., EAKINS P.R. et TIPHANE M., 1962. Shefford map area, Shefford and Brome counties, Eastern Townships of Québec. Ministère des Richesses naturelles, Québec, DPV 187, Québec, Canada, 145 p.

DJINENG NJOMO G. T., 2002. Utilisation des méthodes géophysiques dans l'étude de la contamination des eaux souterraines par les nitrates sur le bassin de la rivière Yamaska. Rapport de stage de maîtrise en Sciences de l'Environnement, Université du Québec à Montréal, Montréal, Canada, 38 p.

GADD N.R., McDONALD B.C. et SHILTS W.W., 1972. Deglaciation of Southern Québec. Commission Géologique du Canada, Rapport 71-47, Québec, Canada, $19 \mathrm{p}$.

GAUDREAU D. et MERCIER M., 1997. La contamination de l'eau des puits privés par les nitrates en milieu rural. Direction de la santé publique de la Montérégie, Longueil, Canada, 64 p.

GIROUX I., 2002. Contamination de l'eau par les pesticides dans les régions de culture de maïs et de soya au Québec Résultats des campagne d'échantillonnage 1999, 2000 et 2001, et évolution temporelle de 1992 à 2001. Ministère de l'Environnement du Québec, Direction des écosystèmes aquatiques, Québec, Canada, 54 p.

HUDAK P.F., 2000. Regional trends in nitrate content of Texas groundwater. J. Hydrol., 228, 37-47.

KATZ B.G., CHELETTE, A.R. et PRATT, T.R., 2004. Use of chemical and isotopic tracers to assess nitrate contamination and ground-water age, Woodville Karst Plain, USA. J. Hydrol., 289, 36-61.

LAROCQUE M., BANTON O. et GAGNON J., 2002. Using models to manage soil inorganic nitrogen in forest tree nurseries. Soil Sci. Soc. Am. J., 66, 602-612.

LASSERRE F., RAZACK M. et BANTON, O., 1999. A GISlinked model for the assessment of nitrate contamination in groundwater. J. Hydrol., 224, 81-90.

MAJDOUB, R. COTÉ, C et DUCHEMIN, M., 2004. Mesures préventives et correctives pour la protection des puits en milieu rural. Vecteur Environ., 37, 31-34.

MCCORMACK R. et LACOULINE M., 1996. Eaux souterraines - état des connaissances. Comptes-rendus Deuxième colloque sur la gestion de l'eau en milieu rural Stratégie de gestion : vers une vision commune, 10-11 sept. 1996, Sainte-Foy, Canada.

McDONALD M. et HARBAUGH A., 1988. A modular 3D finite-difference groundwater flow model. USGS TWRI.

MENV (Ministère de l'Environnement du Québec), 2005. (Page web), Disponible à www.mddep.gouv.qc.ca/eau/ souterraines/sih/index.htm. Consultation en 2004.

MENV (Ministère de l'Environnement du Québec), 2004. Étude de la qualité de l'eau potable dans sept bassins versants en surplus de fumier et impacts potentiels sur la santé. Ministère de l'Environnement du Québec, Québec, Canada.

PARÉ D., 1978. Étude hydrogéologique du bassin de la Yamaska. Ministère des Richesses naturelles, Direction générale des eaux, Québec, Canada, $51 \mathrm{p}$.

POISSON G. 2004. Communication personnelle. MAPAQ (Ministère de l'Agriculture, des Pêcheries et de l'Alimentation du Québec).

POLLOCK D. W., 1994. User's Guide for MODPATH/ MODPATH-PLOT, Version 3: A particle tracking postprocessing package for MODFLOW, the U. S. Geological Survey finite-difference ground-water flow model. Report 94-464, USGS, Reston, Virginia.

PRICHONNET G., 1977. La déglaciation de la vallée du Saint-Laurent et l'invasion marine contemporaine. Géog. Phys. Quat., 31 (3-4), 323-345.

PRICHONNET G., DOIRON A. et CLOUTIER M., 1982. Le mode de retrait glaciaire tardiwisconsinien de la bordure appalachienne, au sud du Québec. Géog. Phys. Quat., 36 (1-2), 125-137.

PRICHONNET G., 1984. Dépôts quaternaires de la région de Granby, Québec - Étude 83-30., Carte au 1:50 000. Commission Géologique du Canada, Ottawa, Canada, 8 p.

PRIMEAU S. et GRIMARD Y. 1989. Rivière Yamaska 1975- 
1988, vol. 2 : Résultats complémentaires sur la qualité des eaux. Ministère de l'Environnement du Québec, Direction de la qualité des cours d'eau, Sainte-Foy, Québec.

REFSGAARD J.C., THORSEN M., JENSEN J.B., KLEESCHULTE S. et HANSEN S. 1999. Large scale modelling of groundwater contamination from nitrate leaching. J. Hydrol., 221, 117-140.

ROBINSON M. A. et REAY W. G., 2002. Ground water flow analysis of a Mid-Atlantic outer coastal plain watershed, Virginia, U.S.A. Ground Water, 40, 123-131.

ROSS M., MARTEL R. PARENT M., LEFEBVRE R. et SAVARD M., 2004. Assessing rock aquifer vulnerability using downward advective times from a 3D model of surficial geology. Comptes rendus GéoQuébec 2004, $5^{\text {e }}$ congrès conjoint SCG/AIH-CNN. Québec, Québec, Canada, 25-27 oct. 2004, 1-8.

SAINT-LAURENT VISION 2000, 1999. L'état du SaintLaurent - La contribution des activités agricoles à la détérioration du Saint-Laurent. Ministère des Travaux publics et Services gouvernementaux du Canada, Québec, Canada.

SIDD (Système d'information sur le développement durable), 2003. Assurer l'avenir du milieu rural : le développement rural et l'agriculture durable au Canada (Page web), Disponible à www.sdinfo.gc.ca/reports/fr/monograph8/ agricult.cfm, Consultation en 2003. 